\title{
GIANT AND MEDIUM CORONARY ANEURYSMS AS A COMPLICATION OF LATE DIAGNOSIS OF KAWASAKI DISEASE: CASE REPORT
}

Angélica Maria Assunção da Ponte Lopes ${ }^{1, *}$, Roberta Oriana Assunção Sousa da Ponte Lopes², Luiz Fernando Pereira de Sá3 ${ }^{3}$ Anna Beatriz Carvalho de Oliveira ${ }^{1}$, Cristiane Patrícia Ferreira Andrade ${ }^{1}$, Pedro Henrique Ferreira Monteiro ${ }^{3}$

1.Centro Universitário UniFacid Wyden, Teresina (PI), Brazil. 2.Hospital Infantil Lucídio Portela, Teresina (PI), Brazil. 3.Centro Universitário Uninovafapi, Teresina (PI), Brazil.

*Corresponding author: angelica.mariaapl@gmail.com

\section{BACKGROUND}

Kawasaki's disease (KD) is an acute inflammatory vasculitis of small and medium vessels, preferentially affects boys under 5 years old and presents as a complication the formation of coronary aneurysms.

\section{CASE REPORT}

Male, 4 years old, had daily high fever and painful cervical adenomegaly, conjunctival hyperemia, oral mucosa and tongue, edema of the extremities and ankle arthritis. Cardiac auscultation: tachycardic, regular rhythm in two stages, normophonetic sounds, without murmur. Tests: Hb 9.8, Ht 29.1, leukocytes 16,200, platelets 552,000, AST 165, ALT153, range GT 574, albumin 2.8, creatinine 0.3, PCR 319, ESR 120, DHL 313, blood cultures and urine culture: negative. Doppler echocardiogram: aneurysmatic dilations of the right (Z-score +15$)$ and left (Z-score +3.3 ) coronary arteries, anterior descending artery (Z-score +19$)$ and circumflex artery (Z-score +9). On the 11th day, after the onset of manifestations, immunoglobulin and acetylsalicylic acid (ASA) were prescribed. Currently, using warfarin and ASA, in outpatient follow-up.

\section{CONCLUSION}

Coronary involvement occurs in 20 to $30 \%$ of untreated patients before 10 days of onset of fever. Early therapy reduces the incidence of coronary aneurysms to $5 \%$. Early diagnosis and treatment lead to a favorable outcome with a reduction in coronary complications. 